Series A

I. MATHEMATICA

493

\title{
FORMAL SERIES, FINITENESS PROPERTIES AND DECISION PROBLEMS
}

BY

A. PAZ

H E L S I N K I 19971

S U O M A L A I N E T I E D A K A TEM I A

doi:10.5186/aasfm.1971.493 
Copyright (C) 1971 by

Academia Scientiarum Fennica

Communicated 11 December 1970 by Arto SalomaA 


\begin{abstract}
Some finiteness properties of formal series over several non-commutative variables are investigated. Some unsolvability results for those properties are proved. A simple example of a context sensitive language over a single letter alphabet which is not probabilistic is exhibited.
\end{abstract}

\title{
Introduction $^{1}$
}

An interesting connection between some classical results in analysis dating back to Cauchy and Jacobi and some quite new theories such as System Theory, Automata Theory and Computability has been established in the past few years. The classical results are concerned with a special type on infinite matrices, called Hankel matrices, connected with the RouthHurwitz problem (see Cantmacher (1959)), with formal series expansion of rational and alg braic functions (see Hurwitz (1889)) and approximations of formal series by an expansion of a rational function - Pade' approximation (see Frobenius (1881)). The formal approach has been rejuvenated recently by Schutzenberger (1961) followed by several others (e.g. Shamir (1967)), Nivat (1968), Flies (1969) who introduced noncommutative variables and studied formal series in connection with the theory of formal languages and automata. Kalman and Ho and others (see Kalman Arbib and Falb (1969)) have applied a generalized form of Hankel matrices to System Theory and solved the realization problem of input-output linear maps. Nasu and Honda, emerging from probabilistic automata theory, have established an interesting finiteness property of a formal series generated by a probablistic automaton, a property which was used by them to prove some undecidebility theorems (see Nasu and Honda (1969)). Finally Carlyle and Paz (1970) have found a connection between a generalized form of Hankel matrices and formal series which are generated by pseudoprobabilistic automata.

The first three sections of this paper are concerned with four finiteness properties of formal series over several noncommutative variables. Those

1) Presented at the Symposium on Probabilistic Automata held at the University of Turku in May, 1970. Research supported in part by Grant 68-1408 at the University of California, Los Angeles. 
properties are related to both classical results and modern ones as mentioned above. While the four properties above where known to be equivalent when a single variable is involved, it is shown that this is no longer true over several noncommutative variables, and the relationship between these properties is investigated in general.

In the next two sections some nonsolvability results connected to the properties described in the first sections are proved, including some open problems left over by Nasu and Honda (1969).

The last section deals with formal languages over a single letter alphabet defined by formal series satisfying finiteness conditions as described in the first sections. A simple example of a context sensitive language which is not probabilistic is presented and it is shown that the problem whether an arbitrary probabilistic language over a single letter alphabet is regular or context-free is not solvable.

\section{A. Word-functions}

Let $X=\langle x, y\rangle$ be a finite alphabet (for the sake of simplicity we shall assume that $X$ contains at most two elements unless otherwise stated), let $X^{*}$ be the free semigroup over $X$ (the set of all "words», or finite sequences of »symbols» or elements, from $X$ including the empty word to be denoted by $A$ ) and let $f$ be a function $f: \Sigma^{*} \rightarrow A$ where $A$ stands for some division ring (in particular $A$ may be equal to $R$ - the set of real numbers which is a field). Such functions will be called »wordfunctions».

Consider the following properties of a given word-function:

(I) For every word $v \varepsilon X^{*}$ there exists an integer $n \leq n_{0}$, where $n_{0}$ is a fixed integer which does not depend on $v$, and elements $C_{n-1}, \ldots$, $C_{0} \varepsilon A$ such that for any two words $u, w \varepsilon X^{*}$ the following equation holds true

$$
f\left(u v^{n} w\right)=C_{n-1} f\left(u v^{n-1} w\right)+C_{n_{-2}} f\left(u v^{n-2} w\right) \ldots+C_{0} f(u w)
$$

where $v^{n}$ is the word $\underbrace{v v v \ldots v}_{n}$ and $u v$ is the catenation of the words $u$ and $v$ (i.e. if $u=x_{1}, \ldots, x_{k}, v=y_{1}, \ldots, y_{j}$ then $u v=x_{1} \ldots x_{k} y_{1}$ $\left.\ldots y_{j}\right)$.

(II) There exists an integer $n, n \times n$ matrices $A(x), A(y)$ over $A$, and $n$-dimensional vectors over $A, \pi$ and $\eta, \pi$ a row vector and $\eta$ a column vector such that for any $w=x_{1} \ldots x_{k} \varepsilon X^{*}$

$$
f(w)=\pi A\left(x_{1}\right) \ldots A\left(x_{k}\right) \eta=\pi A(w) \eta
$$


(III) Let $\triangle(f)$ be the infinite matrix $\triangle(f)=\| a_{i j}(f \|)$ with $a_{i j}(f)=$ $f\left(u_{i} u_{j}\right)$ where $u_{1} u_{2} u_{3} \ldots$ is a fixed preassigned lexicographical ennumeration of the elements of $X^{*}$

$$
\triangle(f) \text { is of finite rank }
$$

(IV) There exists two polynomials $P(x, y)$ and $Q(x, y)$ with coefficients in $A$ and (noncommutative) variables in $X$ such that:

$$
Q(x, y)\left(\sum_{v_{\varepsilon} X^{*}} f(v) v^{-1}\right)=P(x, y) \text { or }\left(\sum_{v_{i} X^{*}} f(v) v^{-1}\right) Q(x, y)=P(x, y)
$$

where the product in the left-hand side is defined in the ordinary way.

The following theorems are either known or can be easily derived from known theorems.

Theorem 1. If $X$ contains a single letter then the four properties (I), (II), (III), (IV) are equivalent for a given word-function.

Proof. That (I) is equivalent to (II) follows as a particular case from a more general theorem proved by Ho (see Kalman Arbib and Falb (1969)). That (I) is equivalent to (III) and (IV) is a classical result which can be found in many books, e.g. Gantmacher (1959) pp. 243-247.

Theorem 2. For the general case ( $X$ contains two or more letters), the properties (II) and (III) are equivalent and both of them imply property (I) for a given word function $f$.

Proof. That (II) is equiralent to (III) follows as a particular case from a more general theorem proved by Carlyle and Paz (1970). That (II) implies (I) has been proved by Nasu and Honda (1968).

\section{B. Some negative results}

Theorem 3. If in property (I) $n \geq 3$ for every $v^{2} \Sigma^{*}$ and $X$ contains two (or more) noncommutative letters (variables) then (I) does not imply (III).

Proof. We shall show how to construct a word function $f$ such that $f$ satisfies (I) but it does not satisfy (III). Let $X=\langle 0,1\rangle$ and consider the following infinite sequence

$$
U=0110100110010110 \ldots=x_{1} x_{2} x_{3} \ldots
$$

constructed as follows: for $x_{i}=0$ or $x_{i}=1$ let $\bar{x}_{i}=1-x_{i}$. The first letter in the sequence is 0 . If the first $2^{k}$ leters (for $k=0,1,2, \ldots$ ) in the sequence have already been fixed and are equal to $x_{1} x_{2} \ldots x_{2^{k}}$ then the next $2^{k}$ letters are $\bar{x}_{1} \bar{x}_{2} \ldots \bar{x}_{2^{k}}$. Let $v, w$ denote finite subsequences of $U$ of the form $v=x_{i} x_{i+1} \ldots x_{i+m}, w:=x_{j} x_{j+1} \ldots x_{j+n}$. It is known that the 
infinite sequence $U$, constructed as above, contains no finite subsequence $v$ such that $v=w w w=w^{3}$ (see Morse and Hedlund (1938)). Consider now the following two sequences of words over $X^{*}$.

$$
\begin{aligned}
& u_{0}=\lambda, u_{1}=x_{1}, u_{2}=x_{1} x_{2}, \ldots u_{i}=x_{1} x_{2} \ldots x_{i} \\
& v_{0}=\lambda, v_{1}=x_{2}, v_{2}=x_{3} x_{4}, \ldots v_{i}=x_{i+1} \ldots x_{2 i}
\end{aligned}
$$

and construct a word function $f, f: X^{*} \rightarrow R$ as follows: $f$ will satisfy a relation of the form (1) with $n \geq 3$ and in addition the following determinants

$$
\left|\begin{array}{l}
f\left(u_{0} v_{0}\right) \ldots f\left(u_{0} v_{i}\right) \\
\vdots \\
f\left(u_{i} v_{0}\right) \ldots f\left(u_{i} v_{i}\right)
\end{array}\right|=\triangle_{i}
$$

will all have nonzero value for $i=1,2,3,4, \ldots$ We prove by induction that the construction of such a function is possible. For $i=1$ we have

$$
\Delta_{1}=\left|\begin{array}{ll}
f(\lambda) & f\left(x_{2}\right) \\
f\left(x_{1}\right) & f\left(x_{1} x_{2}\right)
\end{array}\right|
$$

and the values of $f$ appearing in this determinant can be choosen at will and in a way such that $\triangle_{1} \neq 0$ without affecting the relation (1) to be satisfied by $f$; for the arguments of $f$ in the entries of $\triangle_{1}$ are words of length $\leq 2$ (the »length» of a word $w=x_{1} \ldots x_{k}$, to be denoted $|w|$ is the number of letters in it, i.e. $|w|=k)$. Assume now that all the values of $f$ have been choosen for words $w$ with $|w| \leq 2 i$ such that $f$ satisfies the relation (1) for such words and $\Lambda_{i} \neq 0 . \Delta_{i+1}$ has the form

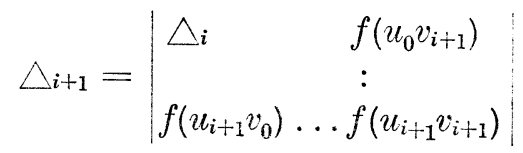

By construction $u_{i+1} v_{i+1}=x_{1} x_{2} \ldots x_{2 i+2}$ wich is an initial subsequence of $U$ of length $2 i+2$ so that, by the property of $U$ mentioned above, the value $f\left(u_{i+1} v_{i+1}\right)$ does not depend on any other values $f(w)$ already fixed - although $f$ is required to satisfy a relation of the form (1). But, by induction, $\Delta_{i} \neq 0$ and therefore, one can choose values $f(w)$ for all $w$ with $|w| \leq 2 i+2$ not yet fixed by the relation (1) including $f\left(u_{i+1} v_{i+1}\right)$ so that $\triangle_{i+1} \neq 0$. It follows that a function $f$ satisfying a relation of the form (1) can be constructed such that $\Delta(f)$ has infinite rank, for the determinants $\triangle_{i}$ are subdeterminants of $\Delta(f)$ for $i=1,2, \ldots$.

Theorem 4. If $X$ contains two or more variables then (IV) does not imply (I) in general and therefore (IV) does not imply (III) or (II) either (for (III) implies (I) by Theorem 2). 
Proof. Let $A$ be the set of real numbers and let $X=\{x, y\}$ where $x$ and $y$ are commutative variables over $A$. Consider the word function $f$ defined as follows:

$$
f(w)=\left\{\begin{array}{l}
1 \text { if } w=x^{m} y^{m} \quad m=0,1, \ldots \\
0 \text { otherwise. }
\end{array}\right.
$$

Then

$$
(x y-1) \sum_{t=0}^{\infty} \frac{1}{x^{i} y^{i}}=x y
$$

Thus $f(w)$ satisfies (IV). But $f(w)$ cannot satisfy (I). To prove this, assume that

$$
f\left(u^{n} v\right)=C_{n-1} f\left(u^{n-1} v\right)+C_{n-2} f\left(u^{n-2} v\right) \ldots C_{0} f(v)
$$

then if $u=x$ and $v=y^{n}$ we must have that

$$
f\left(x^{n} y^{n}\right)=C_{n-1} f\left(x^{n-1} y^{n}\right)+\ldots \ldots+C_{0} f\left(y^{n}\right)
$$

which is impossible for $f\left(x^{n} y^{n}\right)=1$ while $f\left(x^{n-i} y^{n}\right)=0$ for $i=1, \ldots, n$.

Remark: Note that the variables in the example of Theorem 4 are commutative. For noncommutative variables Theorem 4 is no longer true. For noncommutative variables (IV) implies (II) (and therefore also III and $(\mathrm{I})$ ). This follows, as a particular case, from a Theorem of Schutzenberger (1961).

Example: Let $A$ be the set of real numbers and let $X=\{x, y\}$ where $x$ and $y$ are noncommutative variables over $A$. Consider the word function $f$ defined as follows:

$$
f(w)=\left\{\begin{array}{l}
1 \text { if } w=(x y)^{m} m=0,1 \ldots \\
0 \text { otherwise }
\end{array}\right.
$$

then

$$
(x y-1) \sum_{i=0}^{x}(x y)^{-i}=x y
$$

but this function $f(w)$ satisfies also (II) as follows: Let $A(x)=\left(\begin{array}{c}01 \\ 00\end{array}\right) A(y)=$ $\left(\begin{array}{l}10 \\ 00\end{array}\right) ; \pi=(10) ; \eta=\left(\begin{array}{l}1 \\ 0\end{array}\right)$. It is easily verified that $f(w)=\pi A(w) \eta$. Note that this function reduces to the function in Theorem 4 when the variables $x$ and $y$ are commutative. 


\section{Two particular cases}

The previous two theorems show that the range of Theorem 2 cannot be extended to the range of Theorem 1 in general. The purpose of the next two theorems is to show that the range of Theorem 2 can be extended slightly, provided that some additional restrictions are imposed on the specified conditions (I), (II), (III).

Theorem 5. If the function $f: X^{*} \rightarrow A$ is such that the Variables $X$ are commutative and $A$ is a division ring then property (I) implies the property (III) and therefore, by Theorem 2, properties (I), (II) and (III) are equivalent.

Proof. Let $f$ be a word function over a two letter alphabet $X=\{x, y\}$ satistiying the conditions of the theorem and satisfying a relation of the form

$$
f\left(u w^{n} v\right)=C_{n-1} f\left(u w^{n-1} v\right)+\ldots+C_{0} f(u v)
$$

where the constants $n, C_{n-1} \ldots C_{0}$ depend on $w$ only and $n \leq n_{0}$, for some fixed integer $n_{0}$ not depending on $w$. Let $\eta$ be any column in $\angle(f)$ such that

$$
\eta=\left(f\left(u_{1} w\right), f\left(u_{2} w\right) \ldots f\left(u_{k} w\right) \ldots\right)^{T}
$$

where $u_{1} u_{2} \ldots$ is the lexicographic enumeration of the words in $\Sigma^{*}$ corresponding to nonzero rows and $|w| \geq 2 n_{0}-1$. Then either $w=w^{\prime} x^{n_{0}}$ or $w=w^{\prime \prime} y^{n_{0}}$ for the variables are assumed to be commutative. Thus either $\eta=\left(v_{i} x^{n_{0}}\right)^{T} i=1,2, \ldots$ or $\eta=\left(v_{i}^{\prime} y^{n_{0}}\right)^{T} i=1,2, \ldots$ where $v_{i}=u_{i} w^{\prime}$ and $v_{i}^{\prime}=u_{i} w^{\prime \prime}$ and in both cases the column $\eta$ is a linear combination of of previous columns in $\triangle(f)$, because of the relation ( $\overline{5})$.

Theorem 6. Let $f$ be a word function $f: \Sigma^{*} \rightarrow$ A such that $\Sigma=$ $\langle x, y\rangle$ and $f$ satisfies the property (1) with $n_{0} \leq 2$ then $f$ satisfies (III).

Proof. Let $f\left(u_{i}\right)$ be the leading term of the $i-t h$ column of $\triangle(f)$. As $u_{0} u_{1}, \ldots$ is a lexicographic ordering of the words in $\Sigma^{*}, i>j$ implies that $\left|u_{i}\right| \geq\left|u_{j}\right|$. If $\left|u_{i}\right| \geq 4$ then $u_{i}$ is representable in one of the forms $u_{i}^{\prime} x^{2} u_{i}^{\prime \prime}$ or $u_{i}^{\prime} y^{2} u_{i}^{\prime \prime}$ or $u_{i}^{\prime}(x y)^{2} u_{i}^{\prime \prime}$ or $u_{i}^{\prime}(y x)^{2} u_{i}^{\prime \prime}$. This follows from the fact that any word of length 4 which is not equal to $(x y)^{2}$ or $(y x)^{2}$ must have a subword of the form $x^{2}$ or a subword of the form $y^{2}$ (this can be checked by straight forward inspection). It follows from the above observation and from the conditions of the theorem that any column in $\triangle(f)$ whose leading term is $f\left(u_{i}\right)$ with $\left|u_{i}\right| \geq 4$ is a linear combination of two columns with leading terms $f\left(u_{j}\right)$ with $\left|u_{j}\right|<\left|u_{i}\right|$ and this concludes the proof. 


\section{Solvability problems}

This and the following section assume besic knowledge of Automata Theory and Recursive Functions Theory.

Let $f_{1}, f_{2}$ be word functions over the same alphabet $X$ and define the following operations on them:

$$
\begin{aligned}
& f_{3}(u)=\left(f_{1} \vee f_{2}\right)(u)=\max \left(f_{1}(u), f_{2}(u)\right) \\
& f_{4}(u)=\left(f_{1} \wedge f_{2}\right)(u)=\min \left(f_{1}(u), f_{2}(u)\right)
\end{aligned}
$$

Nasu and Honda (1969) considered the following problem: Is it recursively solvable to ascertain for arbitrary word functions $f_{1}$ and $f_{2}$ satisfying the property (II) whether $f_{1} \vee f_{2}$ and $f_{1} \wedge f_{2}$ satisfy that same property?

A negative answer to the above question was given by the above authors using an example as follows: A set of languages $\{L\}(L$ is a set of words $\left.L \subset \Sigma^{*}\right)$ and two sets of word functions $\left\{f_{1}\right\}\left\{f_{2}\right\}$ over an alphabet with two letters are given such that:

(1) It is recursively unsolvable for an arbitrary element $L$ in $\{L\}$ to ascertain whether $L=\varnothing$ ( $\varnothing$ is the empty set of words).

(2) $L=\varnothing$ implies that $f_{1} \vee f_{2}$ satisfies the property (II) where $f_{1} \varepsilon\left\{f_{1}\right\}$ $f_{2} \varepsilon\left\{f_{2}\right\}$ and $f_{1} f_{2}$ are determined by $L$.

(3) $L \neq \varnothing$ implies that $f_{1} \vee f_{2}$ does not satisfy the property (I), $f_{1} f_{2}$ are as in (2). As (II) implies (I) we have that

(4) $L \neq \varnothing$ if and only if $f_{1} \vee f_{2}$ satisfies (II) and therfore it is recursively unsolvable to ascertain for arbitrary $f_{1} \vee f_{2}, f_{1} \varepsilon\left\{f_{1}\right\}, f_{2} \varepsilon\left\{f_{2}\right\}$ whether $f_{1} \vee f_{2}$ satisfies (II) (a similar argument do for $f_{1} \wedge f_{2}$ ). This is Theorem 24 in Nasu and Honda (1969). There are however some additional consequences to be drawn from the above example and our work here. Those consequences are given in the following corollaries.

Corollary 1. For two arbitrary functions $f_{1}$ and $f_{2}$ which satisfy both property (I) or (III), it is recursively unsolvable to decide whether $f_{1} \vee f_{2}$ and $f_{1} \wedge f_{2}$ satisfy (I) or (III), respectively: provided that the alphabet $X$ contains two or more letters.

Proof: (2) and (3) above imply not only (4) but also that:

(5) $L=\varnothing$ if and only if $f_{1} \vee f_{2}$ satisfy (I) (this following from the fact that (II) implies (I) and if $f_{1}$ and $f_{2}$ satisfy (II) they also satisfy $(\mathrm{I})$ ). The statement regarding property (III) follows from Theorem 2.

Remark: As properties (I) and (II) are not equivalent (Theorem 2 and Theorem 3 here) Corollary 1 is not equivalent to the theorem of Nasu and Honda cited above. 
Corollary 2. It is recursively unsolvable to decide for an arbitrary word. function $f$ whether $f$ satisfies property (I) or property (II) or property (III) provided the alphabet $X$ contains two or more letters.

Proof. Unsolvability of property (II follows from the theorem of Nasu and Honda. Unsolvability of (I) follows from Corollary 1 above and unsolvability of (III) follows from Theorem 2 and from the unsolvability of (II).

In the above cited paper of Nasu and Honda (1969) they considered the nonsolvability problems for functions over alphabeta containing at least two letters, leaving the single-letter case open. We shall prove here that the nonsolvability results proved by them are valid also for the single etter case using an example suggested by them and introduced first by this author (Paz, 1966).

\section{E. Unsolvability for single letter alphabets}

We introduce first the following three functions over a single letter: alphabet $\Sigma=\{\sigma\}$.

$$
\begin{array}{r}
f\left(\sigma^{k}\right)=\pi[A(\sigma)]^{k} \eta, k=1,2, \ldots \text { with } \\
\pi=\left(\begin{array}{lll}
0 & 0 & 1
\end{array}\right) ; \eta=\left(\begin{array}{ccc}
\frac{2}{3} & 0 & \frac{1}{3} \\
0 \\
1
\end{array}\right) ; A(\sigma)=\left[\begin{array}{ccc}
\frac{5}{9} & \frac{1}{3} & \frac{1}{9} \\
\frac{1}{4} & \frac{1}{4} & \frac{1}{2}
\end{array}\right]
\end{array}
$$

Thus, $f$ satisfies the condition (II) by its definition.

The following property of $f$ can be proved using an argument similar to the one used in Paz (1966, p. 31):

For any integer $n$ there are integers $t, k_{1}$ and $k_{2}$ such that:

$$
\begin{gathered}
f\left(\sigma^{k 1} \sigma^{n t}\right)>\frac{4}{11} ; \quad f\left(\sigma^{k 2} \sigma^{n t}\right) \leq \frac{4}{11} \\
f\left(\sigma^{k} i \sigma^{p t}\right) \leq \frac{4}{11} ; i=1,2 . \quad p=0,1.2, \ldots, n-1
\end{gathered}
$$

(2) Let $g$ be the function $g\left(\sigma^{k}\right)=\frac{t}{11}$ for $k=1,2 \ldots$ The function $g$ satisfies the condition (II) trivially.

(3) Let $\{Z\}$ be the family of Turing machines. For erery $Z \varepsilon\{Z\}$ define the word function $h_{Z}$ as follows: $h_{Z}\left\{\sigma^{n}\right\}=1$ if $Z$ is still moving after $n$ steps, beginning from blank tape and $h_{Z}\left(\sigma^{n}\right)=0$ otherwise. Then $h_{Z}$ is recursive and total assuming one of the two following forms: 
Either $h_{Z}\left(\sigma^{n}\right)=1$ for $n=1,2 \ldots$

$$
\text { or } h_{Z}\left(\sigma^{n}\right)=0 \text { for } n>n_{0}
$$

(if $Z$ halts after $n_{0}$ steps).

Although it is undecidable to ascertain for arbitrary $h_{Z}$ which of the two forms is the actual form of $h_{Z}$ still in both cases $h_{Z}$ satisfies the condition (II) for any $Z$ as one verifies easily.

Proposition 1. For arbitrary $h_{Z}$ as above the functions $f h_{Z}$ and $g h_{Z}$ satisfy the condition (II).

Proof. It was proved in Paz (1966) that the class of functions satisfying the condition (II) with the additional restriction that the matrices $A(\sigma)$ be stochastic is closed under multiplication. The proof can be extended readily so as to remove the above additional restriction.

Proposition 2. The function $f \vee g$ does not satisfy the condition (II).

Proof: By Theorem 1 it suffices to show that $f \vee g=F$ does not satisfy condition (1). Assume the contrary, then there is some integer $n$ such that for any integer $t$ the function $F$ satisfies a relation of the form

$$
\left.F\left(\sigma^{k} \sigma^{n t}\right)=\sum_{i=1}^{n} C_{n-1} F\left(\sigma^{k} \sigma^{(n-i) t}\right)\right) \quad k=0,1, \ldots
$$

Evaluating this relation first for $t$ and $k_{1}$ as in the remark after the definition of the function $f$ one finds that:

$$
\begin{aligned}
& \frac{4}{11}<\max \left(\frac{4}{11}, f\left(\sigma^{l .1} \sigma^{n t}\right)\right)=F\left(\sigma^{k 1} \sigma^{n t}\right)= \\
& =\sum_{i=1}^{n} C_{n-1} F\left(\sigma^{k 1} \sigma^{(n-i) t}\right)=\sum_{i=1}^{n} C_{n-1} \max \left(f\left(\sigma^{k 1} \sigma^{(n-i) t}\right) \cdot \frac{4}{11}\right)= \\
& =\frac{4}{11} \sum_{i=1}^{n} C_{n-1} \\
& \quad \text { or } \quad \sum_{i=1}^{n} C_{n-1}>\frac{4}{11}
\end{aligned}
$$

On the other hand, evaluating the relation for $t$ and $k_{2}$ one finds in the same way that $\sum_{i=1}^{n} C_{n-1}=\frac{4}{11}$ which is a contradiction.

Theorem 7. It is recursively unsolvable to determine for arbitrary word functions $f, g$ satisfying both the condition (II) and over a single-letter alphabet $\Sigma=\{\sigma\}$ whether $f \vee g$ or $f \wedge g$ satisfy the condition (II).

Proof: We shall prove the Theorem for $f \vee g$, the proof of the other statement being similar.

Consider the functions $f h_{Z}$ and $g h_{Z}$ for arbitrary $h_{Z}$ as exhibited at the beginning of this section. Both of them satisfy the condition (II) (Pro- 
position 1). But the function $f h_{Z} \vee g h_{Z}=(f \vee g) h_{Z}$ does not satisfy this condition if and only if $h\left(\sigma^{n}\right)=1$ for all $n$ (for if $f\left(\sigma^{n}\right)=0$ for $n>n_{0}$, which is the only alternative, then $(f \vee g) h_{z}$ will assume only finitely many nonzero values), which is undecidable by the choice of the set $\left\{h_{z}\right\}$.

Corollary 3. It is recursively unsolvable to determine for an arbitrary given word function over a single letter alphabet whether this function satisfies either of the conditions (I), (II), (III) or (IV).

Proof: Property (II) is undecidable for the function exhibited in Theorem 7 and the other properties are equivalent to property (II) by Theorem 1 .

\section{F. Languages over a single letter alphabet}

Given a word function $f$ we define the languages:

$$
\begin{aligned}
L(f) & =\left\{x: x \varepsilon \Sigma^{*}, f(x) \neq 0\right\} \\
L(f, \lambda) & =\left\{x: x \varepsilon \Sigma^{*}, f(x)>\lambda\right\}
\end{aligned}
$$

where $\lambda$ is a real number.

Languages of the first form have been introduced by Shutzenberger (1961) and languages of the second form have been introduced by Rabin (1963). Let us assume now that the word function $f$ in the definition of the languages above satisfies the property (II). The following properties are well known or easily proved for this case:

(1) There are nonregular languages which can be represented in the form $L(f)$ (Turakainen, 1968).

(2) Every language representable in the form $L(f)$ is representable also in the form $L(f, \lambda)$ ( $f$ in both cases satisfies (II )) but the converse is not true.

For the first part see Turakainen (1968). For the second part consider the language $L=\left\{x^{n} y^{n}: n=1,2, \ldots\right\}$. It has been proved by Turakainen (1969) that it can be represented in the form $L(f, \lambda)$ with $f$ satisfying (II). That it cannot be represented in the form $L(f)$ can be proved. using an argument identical to the argument used in the proof of Theorem 4: If $L(f)=\left\{x^{n} y^{n}: n=1,2, \ldots\right\}=\{w: f(w) \neq 0\}$ then $f$ cannot satisfy property (I), but $f$ is assumed to satisfy property II which implies property (I).

(3) The cardinality of the set of languages representable in the form $L(f, \lambda)$ with $f$ satisfying property (II) is equal to the cardinality of the continuum (Rabin (1963)) which is also the cardinality of the set of all languages ( $\Sigma$ being finite, $\Sigma^{*}$ is countable and the set of all subsets of $\Sigma^{*}$ has the cardinality of the continuum). 
The following problem is now natural. Are there languages not representable in the form $L(f, \lambda)$ with $f$ satisfying (II)? Bukharaev (1965) has given an example of such a language over a single letter alphabet. Unfortunately, he did not prove his example (which is quite complicated, being defined on the basis of four arbitrary primitive recursive functions). We have not been able to prove Bukharaev's result, not have we succeded in establishing contact with him (directly or indirectly) and, to the best of our knowledge nobody else in the west knows how to prove that result (see, e.g. Salomaa who needed that results in order to establish some relation between time variant events and events defined as above). Note that, when looking for a language as above, one must take into consideration that there may be different word functions defining the same language; in addition, if a single letter alphabet is assumed then, the required language if existent must be at least context sensitive, for context free languages over a single letter alphabet are regular and therefore also representable in the form $L(f, \lambda)$ with $f$ satisfying (II) (see Paz (1966)).

We show now an example of a nonrepresentable language over a single letter alphabet.

Let $X=\{x\}$ and let $\triangle=\{a, b\}$. Let $u_{1} u_{2} \ldots$ be a lexicographical enummeration of all nonempty words over $\triangle$ and let $U$ be the infinite sequence of letters from $\triangle$ resulting from the concatenation of the Words $u_{1} u_{2} \ldots$ in their given order. Let $U(i)$ denote the $i$-th letter in $U$ and define the language $L_{1}=\left\{x^{k}: U(k)=a\right\} . L$ is not representable in the form $L(f, \lambda)$ with $f$ satisfying property (II).

Proof: Assume $L_{1}=L(f, \lambda)$ for some $f$ satisfying property (II). As property (II) implies property (I) (Theorem 2 here) there are constants $C_{0} \ldots C_{n-1}$ such that

$$
f\left(x^{k+n}\right)=C_{0} f\left(x^{k}\right)+C_{1} f\left(x^{k+1}\right)=\ldots+C_{n-1} f\left(x^{k+n-1}\right)
$$

Let $\varepsilon_{0} \ldots \varepsilon_{n}, \delta_{0} \ldots \delta_{n}$ be two words in $\iota^{*}$ defined as follows: $\varepsilon_{i}=b$ if and only if $C_{i}>0, \varepsilon_{i}=a$ otherwise, $\varepsilon_{n}=a, \delta_{i}=\Delta-\varepsilon_{i}$. By the construction of $U$ there are integers $k_{1}$ and $k_{2}$ such that:

$$
\begin{aligned}
& U\left(k_{1}\right) \ldots U\left(k_{1}+n\right)=\varepsilon_{0} \ldots \varepsilon_{n} \\
& U\left(k_{2}\right) \ldots U\left(k_{2}+n\right)=\delta_{0} \ldots \delta_{n}
\end{aligned}
$$

It follows from the definitions that:

$$
\begin{aligned}
C_{i}>0 & \Rightarrow U\left(k_{1}+i\right)=\mathrm{b}, U\left(k_{2}+i\right)=a \\
& \Rightarrow f\left(x^{k_{1}+i}\right) \leq \lambda, f\left(x^{k_{2}+i}\right)>\lambda . \\
C_{i} \leq 0 & \Rightarrow U\left(k_{1}+i\right)=a, U\left(k_{2}+i\right)=b \\
& \Rightarrow f\left(x^{k_{i}+i}\right)>\lambda, f\left(x^{k_{2}+i}\right) \leq \lambda .
\end{aligned}
$$




$$
U\left(k_{1}+n\right)=a, U\left(k_{2}+n\right)=b \Rightarrow f\left(x^{k_{1}+n}\right)>\lambda, f\left(x^{k_{2}+n}\right) \leq \lambda .
$$

Evaluating the formula (6) first for $k=k_{1}$ and then for $k=k_{2}$ we have

$$
\lambda<f\left(x^{k_{1}+n}\right)=\sum_{i=0}^{n-1} C_{i} f\left(x^{k_{1}+i}\right) \leq \lambda \sum_{i=0}^{n-1} C_{i}
$$

and

$$
\lambda \geq f\left(x^{k_{2}+n}\right)=\sum_{=0}^{n-1} C_{i} f\left(x^{k_{2}+i}\right) \geq \lambda \sum_{i=0}^{n-1} C_{i}
$$

which is a contradiction.

Remark: It is easily seen that the language $L_{1}$ is context-sensitive for the sequence $U$ can be generated by a linear bounded automation, and, as remarked above, no single letter and simpler language can satisfy the required properties. On the other hand by constructing the sequence $U$ in a more complex way (but still containing as finite subsequences all words in $\triangle^{*}$ ) one can construct languages not representable in the form $L(f, \lambda)$ with $f$ satisfying (II), but not context-sensitive either. This remark is due to $\mathrm{S}$. Greibach.

We conclude this work with the following:

Theorem 8. It is recursively unsolvable to determine for an arbitrary word function $f$ satisfying condition (II) and over a single letter alphabet, and an arbitrary number $\lambda, 0 \leq \lambda<1$, whether the language $L(f, \lambda)$ is regular or whether $L(f, \lambda)$ is context-free.

Proof: Consider the function $f h_{Z}$ as in Proposition 1. $f h_{Z}=f$ if $h_{Z}$ assumes only the value 1 for every argument and $f h_{Z}$ has only finitely many values otherwise. It was proved in $\mathrm{Paz}$ (1966) that $L(f, 4 / 11)$ is not regular. It follows that $L\left(f h_{Z} 4 / 11\right)$ is not regular if and only if $h_{Z}$ assumes only the value 1 which is recursively unsolvable for arbitrary $h_{Z}$. The second satement of the theorem follows from the fact that regular and single-letter larguages are context-free.

Remark 1. Usirg the functions $h_{Z}$ and $1-h_{Z}$ one can prove also that it is recursively unsolvable to decide for an arbitrary word function $f$ satisfying (II) and over a single letter alphabet, and arbitrary number $\lambda$ whether the language $L(f, \lambda)$ is empty or whether it is equal to $\Sigma^{*}$.

Remark 2. Note that the theorems 7 and 8 are existential. The functions $h_{Z}$, on which their proofs are based, are shown to satisfy (II) but their representation in the form (2) is not explicit. One may still ask therefore whether the theorems can be improved by showirg how to construct the functions $h_{Z}$ explicitely in the form (2), for any given $Z$ or else by finding a subset of the set $\{Z\}$ for which this is possible and such that the halting problem from blank tape is still unsolvable for it. This remark 
is specially noteworthy with regard to Theorem 8, for the problem considered in that Theorem is decidable in many cases, provided that the function is given explicitely in the form (2), as follows from the papers of Paz (1966) and Turakainen (1968).

Acknowledgements. The author is indebted to M. O. Rabin for several stimulating discussions.

\section{Computer Science Department}

Technion, Haifa,

Israel

\section{References}

[1] Bukharaev, R. (1965, Criteria for representation of events in fin te probabilistic automata - Dokl Acad. Nauk SSSR, 164, 289-291.

[2] Caryle, J. W. and Paz, A. (1970), "On realization problems for stochastic systems» - Technical report U.C.L.A. Dept. of system sciences.

[3] Fures, M. (1969), Transductions et series formelles, Thesis - Institut Blaise Pascal, Paris.

[4] Froesiss, G. (1881), Über Relationen zwischen Näherungsbrüchen von Potenzreihen - J. Reine u. angew. Math., 90, 1-17.

[5] Gantmacher, F. R. (1959), Applications of the theory of matrices - Intersciences, N.Y.

[6] Hurwitz, A. (1899), Sur un théorème de M. Hadamard - C. R. Acad. Sci. Paris, $128,350-353$.

[7] Kalman, R. E. Falb, P. L. and Arbib, M. A. (1969) - Topics in mathematical system theory, McGraw Hill.

[8] Morse, M. and Heducxd, G. (1938), Symbolic dynamics - Am. J. of Math., 60, $815-866$.

[9] NASU, M. and Hoxda, న. (1968), Fuzzy events realized by finite probabilistic automata - Information and Control, 12, 284-303.

[10] -»- (1969), Mappings Induced by PGSII-mappings and some recursively unsolvable problems of finite probabilistic automata, - Information and Control, 15, 250-273.

[11] Nivat, M. (1968). Transductions des Language de Chomsky - Am. de l'Institut Fourier de L'université de Grenoble. XVIII Fl, 339-455.

[12] PAz, A. (1966), Some aspects of probabilistic automata - Information and Control, 9, 26-60.

[13] RabiN, M. O. (1963) Probabilistic automata - Information and Control, 6, $230-$ 245.

[14] SalomaA, A. (1968), On finite automata with a time-variant structure - Information and Control, 13, 85-98. 
[15] Shamir, E. (1967), A representation theorem for algebraic and context-free power series in non-commutating variables - Information and Control, 11, $239-254$.

[16] Schützenberger, M. P. (1961), On the definition of a family of automata Information and Control, 4, 245-270.

[17] Turakainen, P. (1968), On probabilistic automata and their generalization Ann. Acad. Sci. Fenn., A. I. 429.

[18] - - (1969), On languages representable in rational probabilistic automata Ann. Acad. Sci. Fenn., A. I. 439. 